\title{
Modeling Transitions in Complex Systems by Multiplicative Effect of Temporal Patterns Extracted from Signal Flows
}

\author{
Ezzat G. Bakhoum ${ }^{1}$ and Cristian Toma ${ }^{2}$ \\ ${ }^{1}$ Department of Electrical and Computer Engineering, University of West Florida, \\ 11000 University Parkway, Pensacola, FL 32514, USA \\ ${ }^{2}$ Faculty of Applied Sciences, Politechnica University, Hagi-Ghita 81, 060032 Bucharest, Romania \\ Correspondence should be addressed to Ezzat G. Bakhoum, ebakhoum@uwf.edu
}

Received 2 December 2012; Accepted 10 December 2012

Academic Editor: Carlo Cattani

Copyright (C) 2012 E. G. Bakhoum and C. Toma. This is an open access article distributed under the Creative Commons Attribution License, which permits unrestricted use, distribution, and reproduction in any medium, provided the original work is properly cited.

\begin{abstract}
This study presents a mathematical model based on Fourier decomposition of a sequence of internal signals generated in a complex system by a sequence of external pulses (time series) for characterizing suddenly emerging phenomena as nonlinear transitions. Newly created temporal patterns extracted from internal signal flow (mathematically represented as oscillations with long period) interact as new entities in a multiplicative manner with subsequent pulses from the external time series (already existing entities) in order to generate nonlinear transitions within the system. Such effects are enhanced when the period of external pulses creating new patterns is similar to the settling time of the complex system (this being the condition for an efficient external action). For complex systems where both classical and quantum phenomena generated by external time series are involved, this mathematical model can correctly explain the transition from classical to quantum behaviour (corresponding to a more ordered structure) avoiding typical contradictions generated by analysis performed on transient time intervals or by wave superposition.
\end{abstract}

\section{Introduction}

Nonlinear phenomena generated by an external time series represented by a sequence of pulses applied upon a complex system can be noticed not just for physical structures, but for biological and human systems also. For example, a set of medium-power shocks applied as transverse force upon a crystalline material fixed at both ends can generate significant deformations (possible breaking effect) which can not be always explained as a superposition of individual effects of each pulse. In a similar manner, transitions generated by periodical phenomena in biological systems cannot be analyzed using a linear model, since genetic mutations are often involved (for this reason genetic algorithms were developed). The human behavior is also driven by basic concepts created by repetitive aspects from the environment. 
Some practical aspects were studied in [1], where it was mentioned that workers using traditional tools apply some medium-power shocks (External Time Series) at certain intervals upon a beam fixed at both ends, before the application of a final great-power shock for breaking the material. Each medium-power shock generates specific damping vibrations (Signal Flow) inside the material medium, and the subsequent shock has to be applied right before the annihilation of these damping vibrations by the fluctuations of the external medium (the Noise). Thus, a certain degree of Coherence for the effects of external pulses could be achieved, for the maximum possible value of the amplitude of the fundamental harmonic corresponding to the envelope of the generated vibrations. The mathematical model in this study uses the fundamental harmonic as a factor in a Coherence Function acting as a free term upon linear systems on extended time intervals. However, the practical aspects regarding breaking phenomena require a mathematical model able to generate a certain high intensity effect within a very short time interval, corresponding to just two or three external pulses.

Similar aspects can be noticed at High-Ordered Low Scale phenomena, by analyzing the wave functions with certain frequencies associated to particles represented as phonons (representing the quanta of the harmonic lattice vibrations for high-ordered complex materials). These wave functions can be represented as a sequence of pulses (a Time Serieseach pulse corresponding to an oscillation); through interaction they generate transitions after a certain number of oscillations for the wave-trains of particles involved in interaction, as a Suddenly-Emerging Effect. However, quantum transitions cannot be considered as instantaneous phenomena, because the frequency (an important physical quantity in quantum dynamics theory) requires a certain time interval for an estimation. So it is important to determine some expected values for their frequency and for their corresponding energy not just in a stationary regime, but in a transient regime also (when supplementary vibrations are just generated).

Linear differential equations are not suitable for modelling such aspects. Better qualitative results were obtained using dynamical equations able to generate practical test functions [2] (similar to wavelets [3]) for generating significant delayed pulses (when a free term which corresponds to an external action is added) [4] and for justifying Spatial Patterns appearing in a certain material medium [5]. However, this model cannot explain the effect of a sequence of external pulses when the time interval between these pulses is large enough so as the final effect not be considered a superposition of individual effects of each pulse. For this reason, some specific differential equations based on the Coherence Function between the generated deformation and an alternating cosine input have been taken into consideration [1]. Since this coherence function vanishes if the output is equal to zero, the initial condition should be set at a small nonzero value (the choice of this initial value being not justified). This model has given good qualitative results for modelling the generation of oscillations with different local maximum/minimum values (for second-order differential equations) similar to wavelets analysis of solitary waves [6] and multiscale behaviour (for higher-order differential equations) similar to multi-scale behavior of waves in materials [7]. However, different initial values could lead to different temporal behavior, this being in contradiction with practical aspects of Suddenly-Emerging Phenomena. Moreover, the $\pi / 2$ phase of the external alternating function $\sin (2 \pi t+\phi)=\cos 2 \pi t$ used in that mathematical model is not justified.

For the above reasons, an improved mathematical model based on the Fourier decomposition of internal signals generated in a system by a sequence of external pulses is introduced in this paper. The model is suited for characterizing Suddenly-Emerging 
Phenomena as non-linear transitions in a newly created Quantum Environment, providing conditions for an effcient external action. This model should be also be able to explain specific features of quantum transitions on a very short (transient) time interval.

\section{Carrier-Envelope Temporal Patterns Created by External Time Series}

For a better understanding of basic features of the proposed mathematical model, we analyse the breaking effect of a set of medium-power shocks (External Time Series) applied as transverse force upon a beam (a crystalline structure-High-Ordered System) fixed at both ends when the time interval between these pulses is large enough cannot be explained as a superposition of individual effects of each pulse.

The effect of each pulse in the middle of the beam can be usually represented as

$$
y(t)=\exp (-\alpha t) \sin \left(\omega_{0} t+\phi\right)
$$

which corresponds to the output of a damped second-order system (both $\alpha$ and $\omega_{0}$ are considered to be positive quantities).

The envelope generated by a single pulse is represented by

$$
f(t)=\exp (-\alpha t)
$$

Since the effect of external shocks acting similar to Dirac functions upon the beam at certain time intervals consists in a specific deformation generated inside the material within a very short time interval, we will consider that the effect of different pulses cannot be superposed (the effect of each pulse is cancelled by next one). For a preliminary analysis, the temporal patterns generated by the long period envelope will be analyzed for a sequence of pulses with period set to unity for $\alpha=5, \alpha=1$ and $\alpha=10$, respectively.

If the envelope is represented by

$$
f(t)=\exp (-5 t)
$$

then the time interval beteween two succesive pulses will be approximately equal to the settling time of this function. The long period envelope will be represented by a periodical signal with a certain constant component and an alternating component, as in Figure 1.

If the time constant of the exponential function corresponding to the envelope is increased, so as the envelope generated by a single pulse to be represented by

$$
f(t)=\exp (-t)
$$

then the same time interval beteween two succesive pulses will be smaller than the settling time of the function. Thus the long period envelope will be represented by a sum of a greater constant component and a smaller alternating component as in previous case, as in Figure 2. 


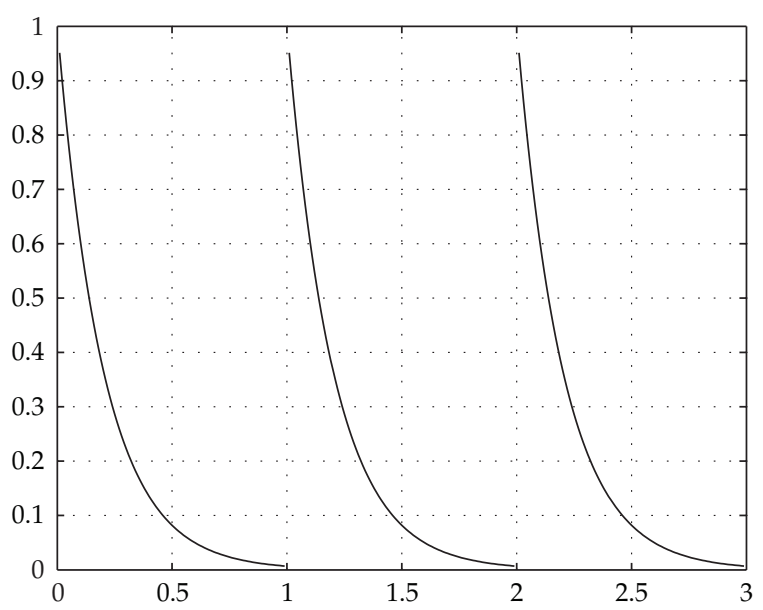

Figure 1: Long period envelope with Period $\approx$ Settling Time.

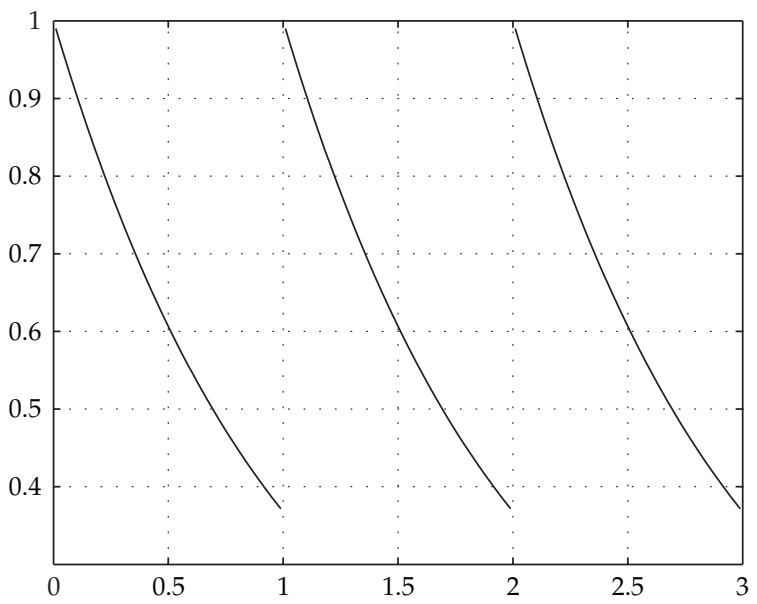

Figure 2: Long period envelope with Period « Settling Time.

If the time constant of the exponential function corresponding to the envelope is decreased, so as the envelope generated by a single pulse to be represented by

$$
f(t)=\exp (-10 t)
$$

then the same time interval beteween two succesive pulses will be greater than the settling time of the function. Thus the long period envelope will be represented by a sum of a smaller constant component and a smaller alternating component as in previous case since the function is approximately zero on large time intervals, as can be noticed in Figure 3.

It can be noticed from the very beginning that a greater alternating component can be obtained for the case when the settling time of the system is comparable to the period between two succesive external pulses.

A more rigorous analysis can be performed using the Fourier decomposition for the long period envelope function. For this purpose, we denote by $T$ the period of 


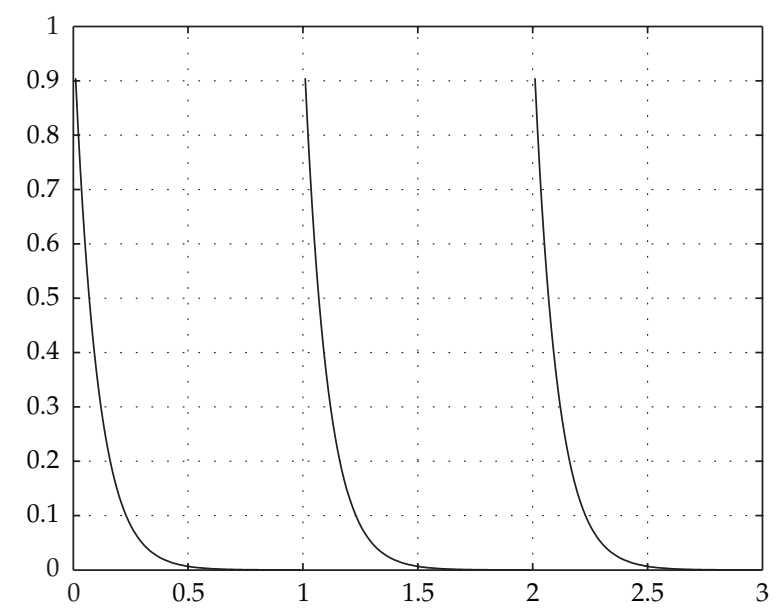

Figure 3: Long period envelope with Period $\gg$ Settling Time.

the external pulses. The analysis will be performed on the time interval $(0,2 \pi / T)$. The constant component does not represent any interest for modellimg non-linear effects. More important is the cosine component of angular frequency $\beta=2 \pi / T$, since transitions are expected to occur when another external shock (usually a great-power shock, as was shown) is applied-this means when the cosine function presents a maximum. The amplitude $A_{\cos }$ of this cosine component will be determined as

$$
A_{\mathrm{cos}}=\frac{1}{T} \int_{0}^{T} \exp (-\alpha t) \cos (\beta t) d t=\frac{\beta}{2 \pi} \operatorname{Re} \int_{0}^{2 \pi / \beta} \exp [(-\alpha+i \beta) t] d t .
$$

It results

$$
A_{\mathrm{cos}}=\frac{\beta}{2 \pi} \operatorname{Re}\left\{\left.\frac{1}{-\alpha+i \beta} \exp [(-\alpha+i \beta) t]\right|_{0} ^{2 \pi / \beta}\right\}
$$

then

$$
A_{\mathrm{cos}}=\frac{\beta}{2 \pi} \operatorname{Re}\left\{\frac{-\alpha-i \beta}{\alpha^{2}+\beta^{2}}\left(\exp \left[(-\alpha+i \beta) \frac{2 \pi}{\beta}\right]-1\right)\right\}
$$

and finally

$$
A_{\mathrm{cos}}=\frac{\alpha \beta}{2 \pi\left(\alpha^{2}+\beta^{2}\right)}\left(1-\exp \left[\frac{-2 \pi \alpha}{\beta}\right]\right) .
$$

By denoting the ratio between $\beta$ and $\alpha$ as $k$ and substituting $\beta=k \alpha$ in previous equation, it results after simplifying certain factors

$$
A_{\mathrm{cos}}=\frac{k}{2 \pi\left(1+k^{2}\right)}\left(1-\exp \left[\frac{-2 \pi}{k}\right]\right) .
$$




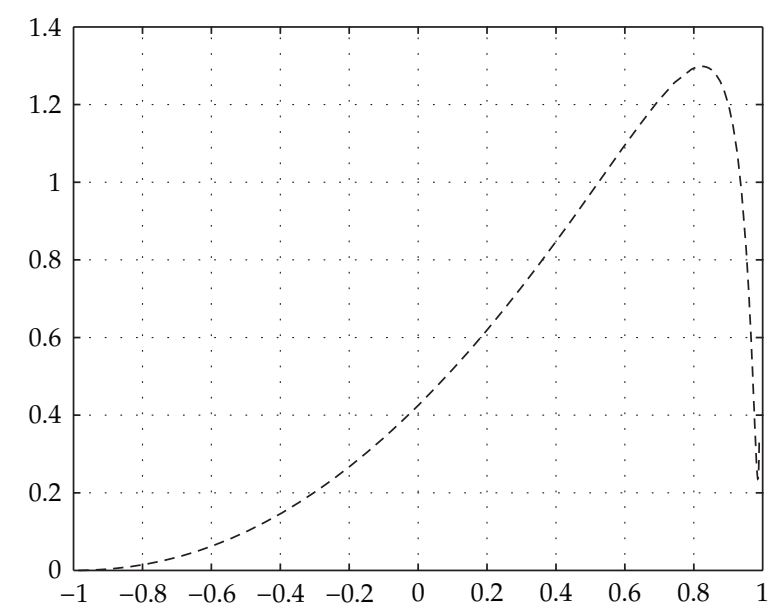

Figure 4: Amplitude of cosine function versus $k$.

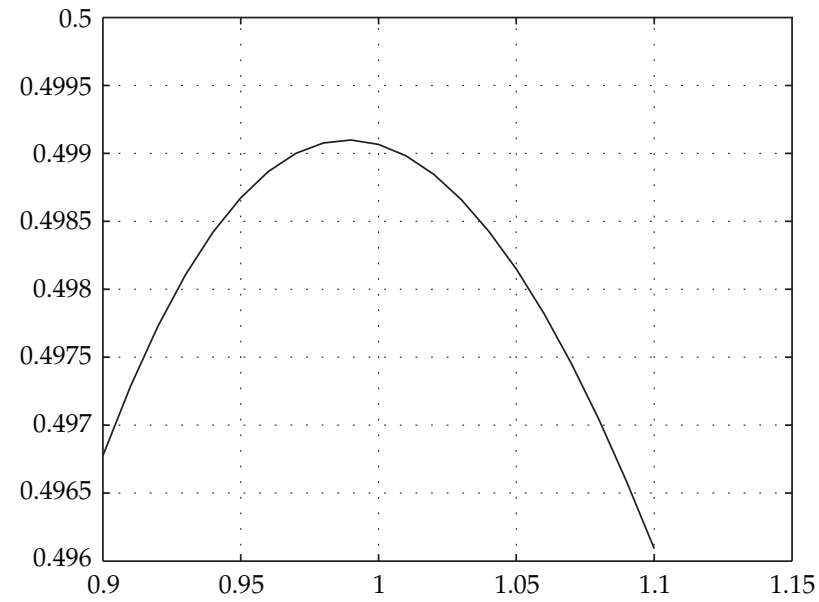

Figure 5: Amplitude of cosine function versus $k$-Details around $k=1$.

This function has zero limit when $k$ trends to zero and to $\infty$, presenting a maximum value for $k \approx 1$. The graph of this function is represented in Figure 4 , for $k \in(0.5,1.5)$ (the $2 \pi$ factor at denominator was not taken into consideration).

It can be noticed that the maximum value is reached for $k \approx 1$. The value of $A_{\cos }$ varies with less than $10 \%$ for a wide range of $k(k \in(0.6,1.5))$. A more refined graph is presented in Figure 5. It can be noticed that the value of $A_{\cos }$ varies with less than $0.6 \%$ for $(k \in(0.9,1.1))$.

The maximum value of $A_{\cos } \approx 0.499 / 2 \pi$ corresponds to $k \approx 0.98$ (the $2 \pi$ factor has been omitted in previous figures). This result was expected, since the factor $k /\left(1+k^{2}\right)$ (with a maximum for $k=1$ ) is multiplied by $1-\exp (-2 \pi / k)$ (a function increasing from 0 to 1 with a small slope for a wide range of values). The physical meaning of this result consists in the fact that a high-amplitude cosine component is obtained when the time period $T$ between two subsequent pulses (depending on $\beta$ as $T=2 \pi \beta \approx 6.28 \beta$ ) is approximately equal to the transient (settling) time $T_{\text {tr }}$ of the damped second order system upon these pulses act 
( $T_{\text {tr }}$ depending on $\alpha$ as $T_{\text {tr }}=4 \ldots 6 \alpha$, according to basic dynamical aspects of second order linear systems). This means that short-period oscillations generated inside the system by an external pulse are not completely damped when another external pulse is applied (usually these short period oscillations are damped by friction phenomena or thermal interaction with the environment).

A slightly different result would have been obtained if the ratio $k$ is adjusted for a maximum amplitude $A_{\text {sin }}$ for the sine component of period $T$ for the long period envelope. In a similar manner, it is determined as

$$
A_{\sin }=\frac{1}{T} \int_{0}^{T} \exp (-\alpha t) \sin (\beta t) d t=\frac{\beta}{2 \pi i} \operatorname{Im} \int_{0}^{2 \pi / \beta} \exp [(-\alpha+i \beta) t] d t .
$$

It results

$$
A_{\text {sin }}=\frac{\beta}{2 \pi i} \operatorname{Im}\left\{\left.\frac{1}{-\alpha+i \beta} \exp [(-\alpha+i \beta) t]\right|_{0} ^{2 \pi / \beta}\right\}
$$

then

$$
A_{\text {sin }}=\frac{\beta}{2 \pi i} \operatorname{Im}\left\{\frac{-\alpha-i \beta}{\alpha^{2}+\beta^{2}}\left(\exp \left[(-\alpha+i \beta) \frac{2 \pi}{\beta}\right]-1\right)\right\}
$$

and finally

$$
A_{\sin }=\frac{\beta^{2}}{2 \pi\left(\alpha^{2}+\beta^{2}\right)}\left(1-\exp \left[\frac{-2 \pi \alpha}{\beta}\right]\right)
$$

Substituting $\beta=k \alpha$ in previous equation, it results

$$
A_{\text {sin }}=\frac{k^{2}}{2 \pi\left(1+k^{2}\right)}\left(1-\exp \left[\frac{-2 \pi}{k}\right]\right)
$$

The ratio between $A_{\text {cos }}$ and $A_{\text {sin }}$ equals $1 / k$. It corresponds to the phase $\Phi$ of the alternating compoment of period $T$ as

$$
\Phi=\arctan \frac{A_{\mathrm{cos}}}{A_{\mathrm{sin}}}=\frac{1}{k}
$$

For $k \approx 1$ (as previously determined) the ratio will be around unity, so $A_{\cos } \approx A_{\text {sin }}$ and $\Phi \approx \pi / 4$. The modulus $A$ of the alternating component of period $T$ can be determined as

$$
A=\sqrt{A_{\mathrm{cos}}^{2}+A_{\mathrm{sin}}^{2}}=\frac{k}{2 \pi \sqrt{1+k^{2}}}\left(1-\exp \left[\frac{-2 \pi}{k}\right]\right) .
$$

However, the transition created by this long period Carrier-Envelope Function is determined (according to our previous assumption) by cosine function, since it is generated around 
zero moment of time (with time origin considered when an external pulse is applied). For this reason the value of $k$ corresponding to the maximum for $A_{\cos }(k \approx 1)$ and an angular frequency $\beta \approx \alpha(1 / \alpha$ being the time constant of the system subject to external pulses) should be taken into consideration for modelling non-linear transitions. The cosine component of this angular frequency could be considered as a Multiplicative Factor for the intensity $I_{\text {pul }}$ of an external pulse, generating a supplementary non-linear effect $E_{\text {non-lin }}$

$$
E_{\text {non-lin }}=A_{\text {cos }} I_{\text {pul }}
$$

which is added to the linear effect $E_{\text {lin }}$ of a single (isolated) pulse

$$
E_{\text {lin }}=k I_{\text {pul }}
$$

so as to result the global effect $E$ of a subsequent pulse in a certain sequence as

$$
E=E_{\text {lin }}+E_{\text {non-lin }}=k I_{\text {pul }}+A_{\text {cos }} I_{\text {pul }}=\left(k+A_{\text {cos }}\right) I_{\text {pul }} \text {. }
$$

It can be noticed that this long period Carried-Envelope Function enhances a certain external pulse generating the transition, for example, the third pulse in a sequence is Multiplied by the envelope cosine component of period $T=2 \pi / \beta$ generated by the effect of two previous pulses. It results that an efficient external action consists in a set of external pulses (a time series) with period similar to the transient time of the complex system where transitions should be generated.

For more complex dynamical models based on Coherence Function where delayed effects and multiscale phenomena appear $[1,8]$, the Fourier decomposition presented in this paragraph justifies the amplitude of the alternating components generated by external pulses taken into consideration for determining the Coherence Function.

\section{Temporal Patterns Created and Self-Organization by Fast-Varying External Signals in Low Scale Physical Systems: Modelling Transition to Quantum Behaviour}

The multiplicative model of long period Carrier-Envelope Function is suitable for modelling and explaining a large class of phenomena in complex physical systems subject to fastvarying external signals when the selection of classical or quantum approach is not obvious for determining expected values through measurement procedures for certain physical quantities.

First we analyze some aspects connected with field emission for charged particles moving on repetitive closed-loop trajectories. When a beam of charged particles moves first time along such a trajectory, then for the first part of the movement (before the particle to return around its initial position) the trajectory should be considered as an open-loop trajectory. According to basic electromagnetic aspects, the particle should radiate energy. After a certain transient time, it can be considered that the particle is in a steady-state which should be described by quantum physics laws, and thus no signal will be emitted anymore. The multiplicative model of Carrier-Envelope Pattern is recommended for modelling the particle behaviour on this transient time, determining expected values for measurement 
procedures in a more rigorous manner. It requires just a few periodical movements in order for a certain amplitude of an alternating component to be determined-then it is multiplied by the potential energy so as a specific term to be added in Schroedinger equation (the need of external fields generating potential energy has been shown in [9] due to the paradox of phase loss for a quantum particle moving in a quantified electrostatic potential). Thus the system achieves a high-ordered structure by itself- through Self Organization based on hiding dynamics and internal memory (see $[10,11]$ for more details about learning hiding dynamics and [12] for systems with memory at biological level of organization). In a certain point of space $M_{S}$, we can consider that after a few periodical movements (when classical laws for observable physical quantities are valid) the set of waves emitted by the particle from different points of space $S_{i}$ along the steady-state trajectory will create also a very low-amplitude Temporal Pattern (a low-amplitude Carrier-Envelope Function), since the phase of received waves presents a circular uniform distribution (practically the coherent waves cancel each other). Thus no emitted field can be detected when the particle is in a steady-state (when the transient time has passed), according to quantum physics laws. This aspect differs to reception of continuous signals based on well-known detection of CarrierEnvelope Functions, where non-linear signal processing is achieved trough additional devices (rectifier diodes, threshold circuits, memories). The use of advanced mathematical aspects as multifractional formula [13], cyber-physical laws [14] and stochastic bound [15] imply also the use of such additional devices for memory and decision.

In a similar manner, when the particle performs a transition from a steady-state to another, a wave train corresponding to a photon is emitted. The wave-train corresponding to this emitted photon is detected by a certain material medium as a non-linear phenomenon, some alternances corresponding to the photon creating a Temporal Pattern enhancing the effect of subsequent alternances and generating the quantum interaction with the receiving material medium.

Some basic features of this model can be extended to analysis of light signals propagating in fluctuating weak gravitational fields. As was shown in [16], in every point in a space, at a certain moment of time should be defined a certain material reference system which acts upon a received light wave-train through Lorentz transformation (thus determinig expected values for space-time measurements based on light wave-trains). In case of vacuum, the last material medium that light has passed through should be taken into consideration. When the light passes near great material bodies, it interacts with the significant gravitational field of that body and thus the reference system of this material body should be taken into consideration. Yet in the limit case, when the gravitational field is approximately zero, some fluctuations created by different material bodies still exist. So the propagating wave can be considered as subject to a great number of Lorentz transformations which could scatter the wave, and the wave should vanish within a short space-time interval (considered at the scale of cosmic systems). Applying the Carrier-Envelope Model, it results that the stochastic lowamplitude variations of the gravitational field around the null value creates a null temporal pattern which does not allow an influence upon a propagating wave-train (low-amplitude vectors with different directions cancel each other). This is in accordance with the fact that, according to Conformal Field Theory, all quantum field theories are only effective over some range of length and energy scales, and those used in particle physics are no more fundamental than in condensed matter.

Dynamical relativistic aspects can be also justified by a Multiplicative Model. The factor $1-v^{2} / c^{2}$ appearing at denominator in mass formula can be obtained just by multiplying $(c-v) / c$ and $(c+v) / c$-as two functions are multiplied. For a movement along $O x$ axis, it 
can be easily understand that when $v$ equals $c$ no interaction between wave and particle occur and thus the mass seems to be infinite (no result of the wave action upon the particle being noticed, so the particle seems to be out of the range of any possible external command). However, according to well-known mass formula the same result is valid when $v=-c$, this means when the particle is moving with light speed $c$ in the opposite direction as related to the received wave. This aspect cannot be explained as lack of interaction between wave and particle (or, generally speaking, as lack of interaction between an external command and the system upon this command acts), and thus a model based on a multiplication of certain functions is necessary. In an intutive manner, these two functions can be put in correspondence with a direct and a reverse wave which interact in the point of space where the particle with mass $m$ is situated (the system and the external command interacts in a more complex manner, as in a sequence action-reaction defined on a very short time interval).

\section{Conclusions}

This study has presented a mathematical model based on Fourier decomposition of internal signals created in a complex environment by a sequence of external pulses for justifying suddenly-emerging phenomena as non-linear transitions. Newly created temporal patterns (mathematically represented as long period oscillations) interact in a multiplicative manner (as a Carrier-Envelope Function) with subsequent pulses, generating supplementary effects which can be noticed both at macroscopic and quantum (microscopic) scale, for physical, biological and human systems. Such effects are enhanced when the period of external pulses is similar to the settling time for the environment, justifiying the amplitude of alternating components used in more elaborated mathematical models based on Coherence Function for simulating suddenly-emerging phenomena. Adding phase aspects, this mathematical model can explain transitions between classical and quantum behaviour in a complex material environment in a correct manner, avoiding contradictions generated by analysis performed on transient time intervals or by wave superposition.

\section{References}

[1] E. G. Bakhoum and C. Toma, "Dynamical aspects of macroscopic and quantum transitions due to coherence function and time series events," Mathematical Problems in Engineering, vol. 2010, Article ID 428903, 13 pages, 2010.

[2] G. Toma, "Practical test-functions generated by computer algorithms," in Proceedings of the International Conference on Computational Science and Its Applications (ICCSA '05), vol. 3482 of Lecture Notes in Computer Science, pp. 576-584, May 2005.

[3] C. Cattani, "Harmonic wavelets towards the solution of nonlinear PDE," Computers and Mathematics with Applications, vol. 50, no. 8-9, pp. 1191-1210, 2005.

[4] B. Lazar, A. Sterian, St. Pusca et al.," "Simulating delayed pulses in organic materials," in Proceedings of the Computational Science and Its Applications (ICCSA '06), vol. 3980 of Lecture Notes in Computer Science, pp. 779-785, 2006.

[5] F. Doboga, "Different structural patterns created by short range variations of internal parameters," in Proceedings of the 7th international conference on Computational Science (ICCS '07), vol. 4488 of Lecture Notes in Computer Science, pp. 1060-1066, 2007.

[6] J. J. Rushchitsky, C. Cattani, and E. V. Terletskaya, "Wavelet analysis of the evolution of a solitary wave in a composite material," International Applied Mechanics, vol. 40, no. 3, pp. 311-318, 2004.

[7] C. Cattani, "Multiscale analysis of wave propagation in composite materials," Mathematical Modelling and Analysis, vol. 8, no. 4, pp. 267-282, 2003.

[8] E. G. Bakhoum and C. Toma, "Specific mathematical aspects of dynamics generated by coherence functions," Mathematical Problems in Engineering, vol. 2011, Article ID 436198, 10 pages, 2011. 
[9] E. G. Bakhoum and C. Toma, "Mathematical transform of traveling-wave equations and phase aspects of quantum interaction," Mathematical Problems in Engineering, vol. 2010, Article ID 695208, 15 pages, 2010.

[10] C. Cattani and A. Ciancio, "Hybrid two scales mathematical tools for active particles modelling complex systems with learning hiding dynamics," Mathematical Models and Methods in Applied Sciences, vol. 17, no. 2, pp. 171-187, 2007.

[11] C. Cattani, A. Ciancio, and A. d'Onofrio, "Metamodeling the learning-hiding competition between tumours and the immune system: a kinematic approach," Mathematical and Computer Modelling, vol. 52, no. 1-2, pp. 62-69, 2010.

[12] A. Ciancio, "An approximate evaluation of the phenomenological and state coefficients for viscoanelastic media with memory," U.P.B. Scientific Bulletin A, vol. 73, no. 4, pp. 3-14, 2011.

[13] M. Li and W. Zhao, "Quantitatively investigating locally weak stationarity of modified multifractional Gaussian noise," Physica A, vol. 391, no. 24, pp. 6268-6278, 2012.

[14] M. Li and W. Zhao, "Visiting power laws in cyber-physical networking systems," Mathematical Problems in Engineering, vol. 2012, Article ID 302786, 13 pages, 2012.

[15] M. Li and W. Zhao, "Representation of a stochastic traffic bound," IEEE Transactions on Parallel and Distributed Systems, vol. 21, no. 9, pp. 1368-1372, 2010.

[16] E. G. Bakhoum and C. Toma, "Relativistic short range phenomena and space-time aspects of pulse measurements," Mathematical Problems in Engineering, vol. 2008, Article ID 410156, 20 pages, 2008. 


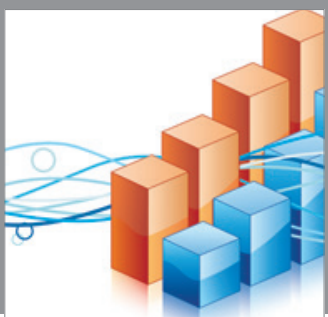

Advances in

Operations Research

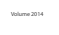

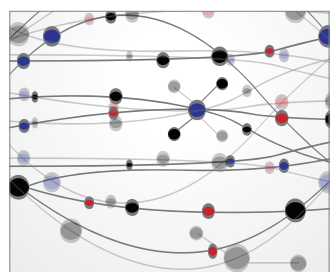

\section{The Scientific} World Journal
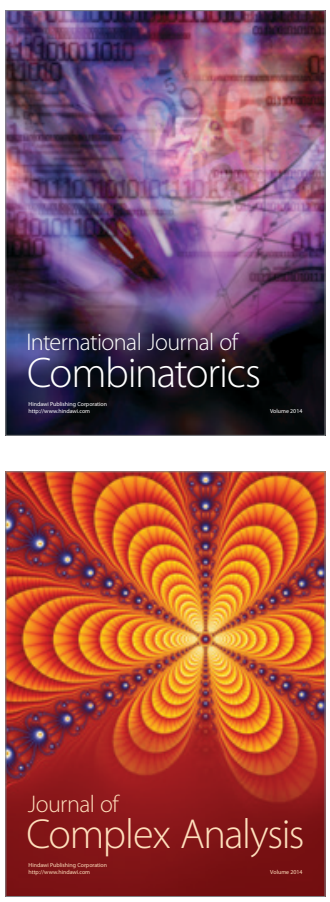

International Journal of

Mathematics and

Mathematical

Sciences
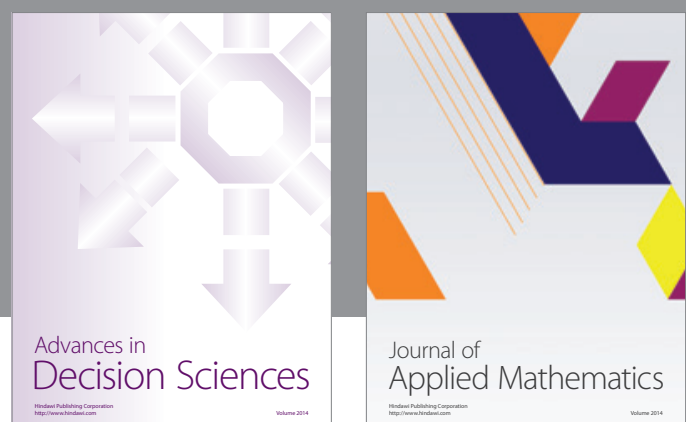

Journal of

Applied Mathematics
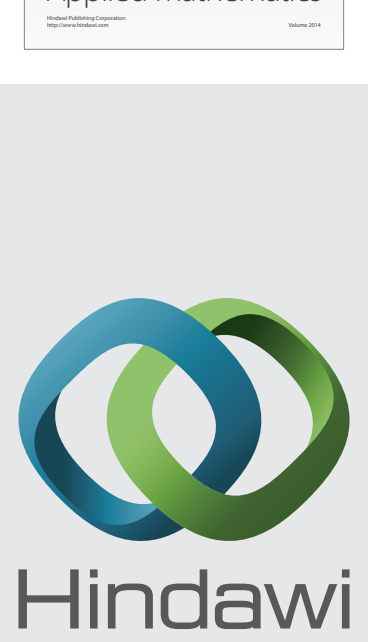

Submit your manuscripts at http://www.hindawi.com
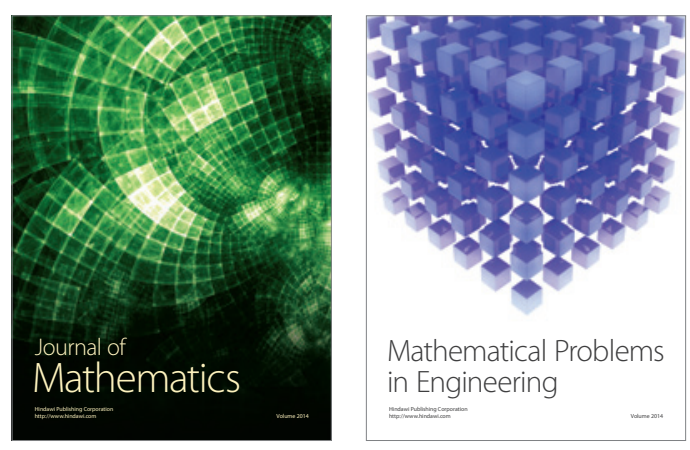

Mathematical Problems in Engineering
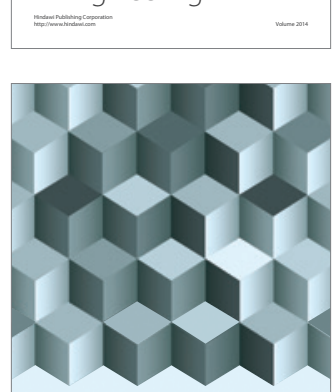

Journal of

Function Spaces
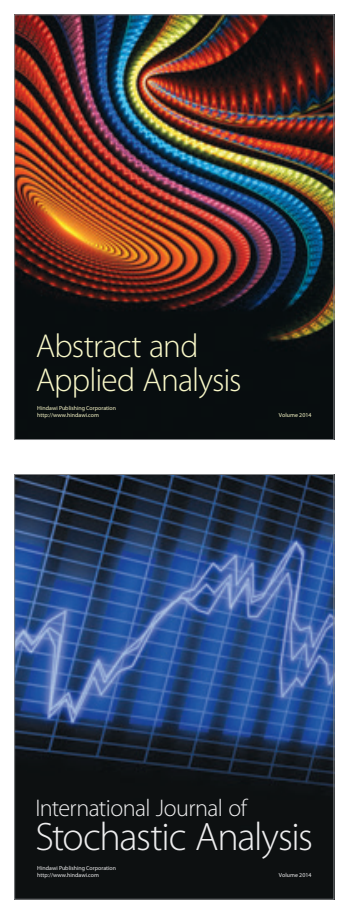

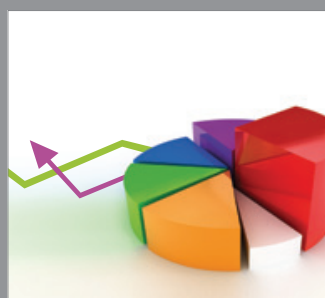

ournal of

Probability and Statistics

Promensencen
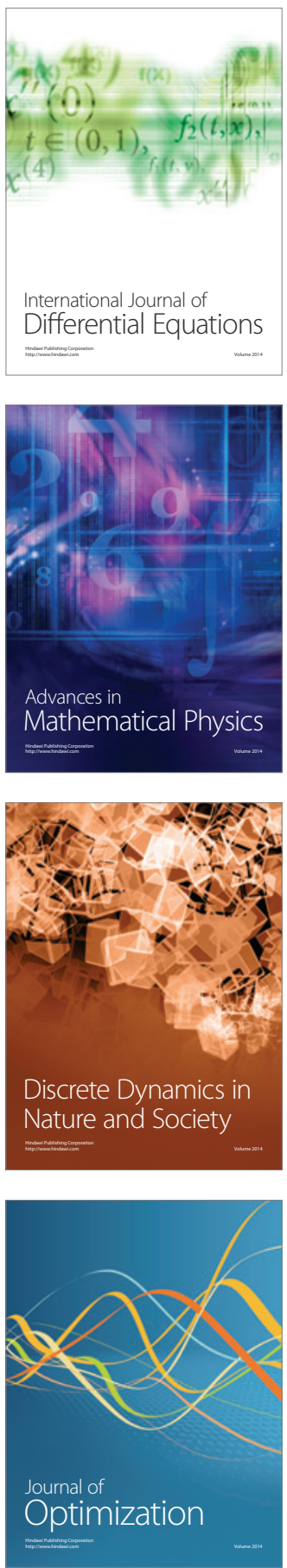